\title{
Case Report of a High Grade Bilateral Thalamic Glioma in an 8Year Old Child Presenting in Psychiatry OPD
}

\author{
Monu Doley ${ }^{1}$ and H Anjuman Choudhury ${ }^{2 *}$ \\ ${ }^{1}$ Postgraduate Trainee, Department of Psychiatry, Silchar Medical College and Hospital, India \\ ${ }^{2}$ Registrar, Department of Psychiatry, Silchar Medical College and Hospital, India \\ *Corresponding Author: Anjuman Choudhury, Registrar, Department of Psychiatry, Silchar Medical College and Hospital, India.
}

Received: July 08, 2019; Published: July 19, 2019

DOI: $10.31080 /$ ASNE.2019.02.0083

\begin{abstract}
Thalamic gliomas are extremely rare neoplasm of the brain. Bilateral thalamic gliomas are even rarer compared to unilateral thalamic tumor. Thalamic glioma though benign in nature but has poor prognosis. We report here a case of high grade bilateral thalamic glioma in an 8year old female child presenting in a psychiatric menifestations like withdrawn behaviour, low mood, excessive daytime sleepiness and a history of sleep walking. The child had a bilateral thalamic bulky mass grossly compressing the third ventricle and protruding into body of lateral ventricles. And MR spectroscopy revealed significant elevation of choline and reduction of NAA.
\end{abstract}

Keywords: Bilateral Thalamic Glioma; High Grade; Excessive Somnolence; Withdrawn Behaviour; Low Mood

\section{Introduction}

A thalamic glioma, as its name implies, is a glial tumour characterized by a primary growth center within or very near the thalamus. It accounts for $1-1.5 \%$ of all brain tumour occurring in children, of which, $25 \%$ are reported in children less than $15 y e a r s$ of age $[3,6]$. Since thalamic glioma are complicated tumours in a declicate area, they are best diagnosed and treated at a major medical center. Bilateral thalamic glioma (BLTG) are extremely rare compared to unilateral glioma. As compared to unilateral thalamic glioma, though tumour are of benign nature but as compared to unilateral thalamic glioma, BLTG has unfavourable outcome. We report a case of high grade bilateral thalamic glioma in a 8year old female child presenting with predominant psychiatric menifestations.

\section{Case Report}

An 8year old female child presented to psychiatry OPD with withdrawn behaviour, low mood, excessive daytime sleepiness and a history of sleep walking. There is also unstable gait, lethargic, puffiness of face for 4 months.
On detailed evaluation, child was born of non-consanguinous marriage with uneventful birth history and normal developmental milestones. At 6 years of age, she had one episodes of febrile convulsion followed by history of sleep walking, intermittent episodes of vomiting, lethargy, dizziness. The symptoms start progressing with gradual deterioration in her physical and mental activities, so she dropped out school one and a half year back. For few months she was relieved symptomatically on treatment for vomiting and general weakness from pediatrician. But as days progressed, child used to remain aloof, not interacting much, decreased oral intake, drowsy and had occasional headache and urinary incontinence, for which he was referred to our Psychiatry side.

- General physical examination revealed: Child is conscious but drowsy with unstable gait. Pallor present and her pupils normal in size, reacting sluggishly to light.

- Mental Status Examination revealed: Dress and hygiene maintained, decreased psychomotor activity, speech is monotonous, low volume and in replying in 1 or 2 words Affect is apathetic, constricted but no formal 
thought disorder or perceptual abnormality seen. Rest of the MSE could be done as patient was drowsy and inattentive.

- Neurological examination revealed: Unsteady gait, bilateral papilloedema and no sensorimotor deficit.

\section{Investigations}

- $\quad$ EEG revealed- sharp and slow wave discharges suggestive of cortical dysrhythmia.

- $\quad$ MRI Brain revealed - Bilateral thalamus which are grossly bulky and lobulated with heterogenous MR signal intensities. Multiple small areas of breakdown are seen in the both thalami. Bulky thalami are causing compression of third ventricle and protruding into the body of lateral ventricles. Lateral ventricles are dilated (Figure 1 and 3).

- The lesion is causing minimal mass effect on the superior surface of tegtum and tegmentum (Figure 4).

- On post contrast study, patchy areas of enhancement are seen on enlarged thalami (Figure 5 and 6).

- On MR Spectroscopy, evidence of significant elevation of choline and reduction of NAA noted (Figure 7). The areas of breakdown shows lactate peak, suggestive of necrosis.

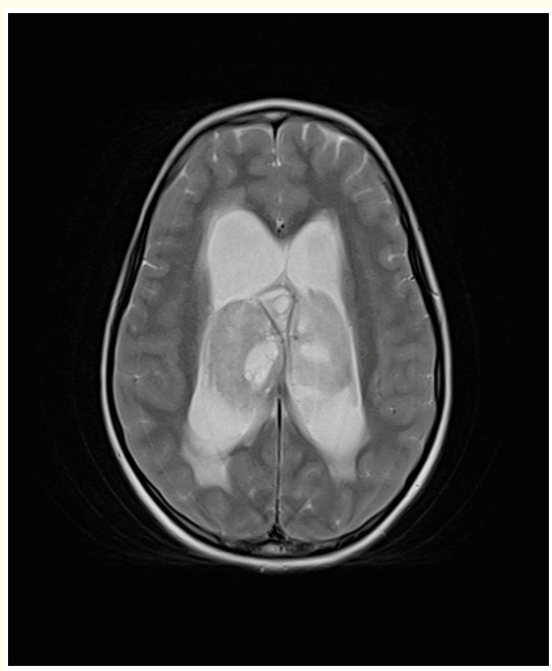

Figure 1: MR Scan of head Axial T2W1 Image showing bilateral thalamic glioma with ventriculomegaly.

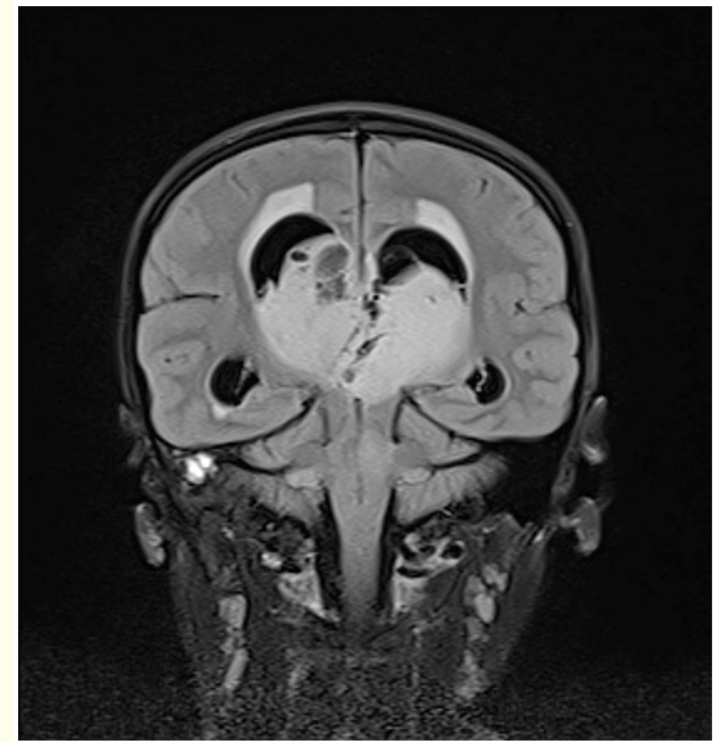

Figure 2: MR Scan of head FLAIR coronal section showing limitation of tumour to thalamus.

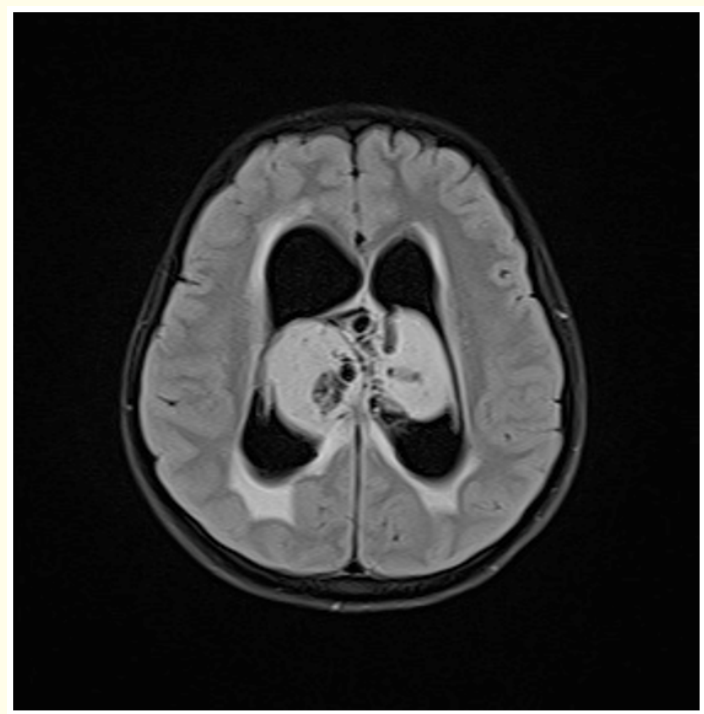

Figure 3: MRI Brain of head FLAIR Axial section showing lucency of periventricular areas suggestive of hydrocephalus. 


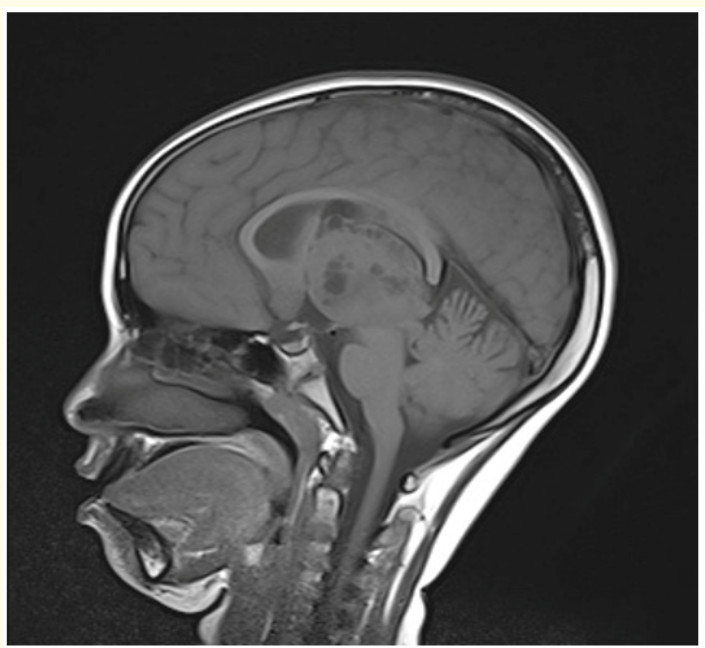

Figure 4: MRI Brain T1W Saggital section showing extension of tumour to midbrain.

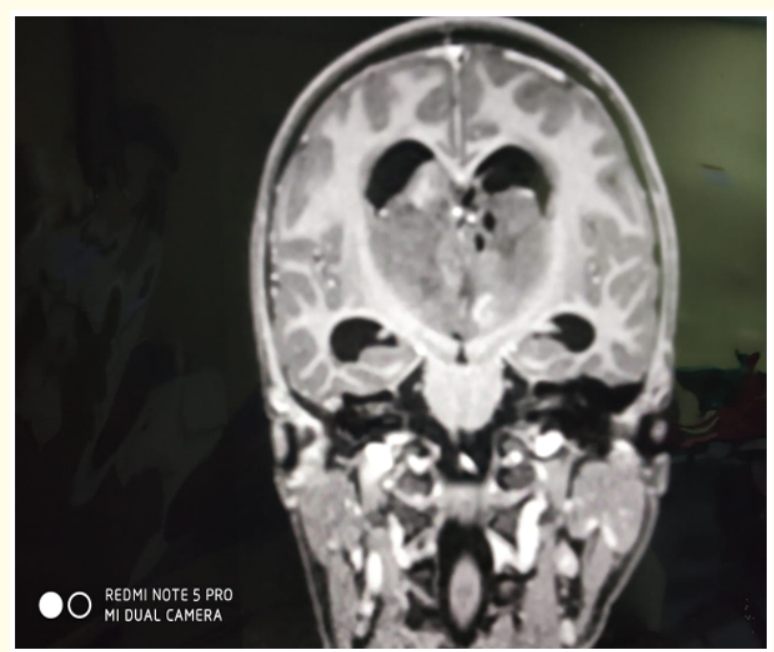

Figure 5: T1W Post contrast image on coronal section showing patchy areas of enhancement on enlarge thalami.

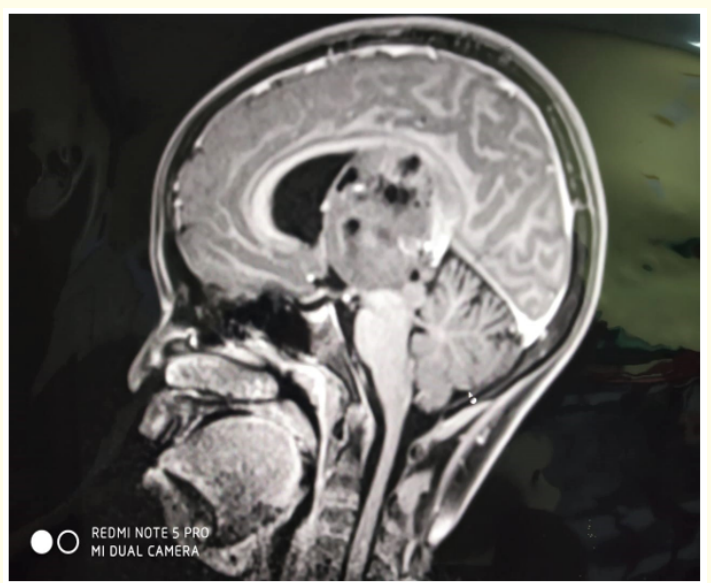

Figure 6: T1W post contrast image of Head on saggital section showing patchy areas of enhancement on larged thalami.

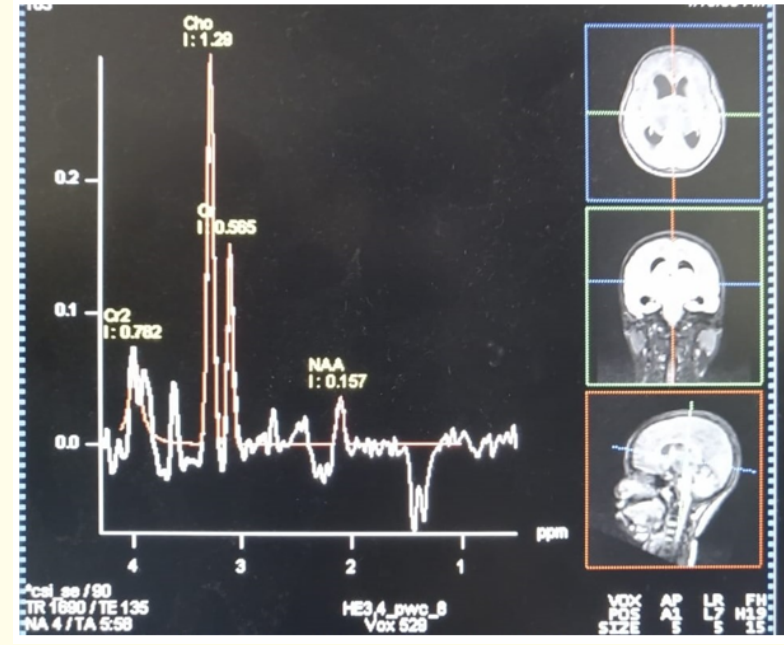

Figure 7: MRS of patient showing choline peak.

\section{Discussion}

Primary bilateral thalamic glioma are uncommon. Most of them are low grade astrocytomas $[11,12]$, in contrast to our case which is high grade astrocytoma. Age of presentation varies from 3months to 80 years [3,12]. No gender predilection more than $50 \%$ of reported case are in paediatric age group [12]. As glioma are of glial cells origin, one school of thoughts is that, glial cells are present through out the CNS. So, glial cells present in the adjacent structure of thalamus ( $3^{\text {rd }}$ ventricle, lateral ventricles, midbrain, pineal gland) may develop into glioma and proliferate to the thalamus. While others consider them to originate from subependymal region of third ventricles $[3,9,11]$. Another thought, two thalami are interconnected through fibres that travel across the midline, this leads to frequent cross-over involving the both sides of thalamus. The clinical presentation of BLTG can be explained when the tumour begins to compress the adjoining ventricles, resulting in CSF outflow blockage. This phenomenon causes headache, vomiting, unstable gait etc. Hydroeplaus are usually absent or mild [2], in contrast to our case where there is prominent hydrocephalus. Fibre tracts surrounding the thalamus when interfered manifest as gait instability, motor weakness, tone abnormalities, nystagmus. As thalamus has role in sleep stages regulation, consciousness and awareness, thalamic glioma alters this functions. In our case, child came to us primarily for excessive day time sleepiness, mild unstable gait. On detailed evaluation it was also seen that she complained of occasional headache, remained withdrawn and had a low mood. Usually affected adults and elderly presents with personality changes, memory deficits, cognitive decline [11]. While in peadiatric population rarely presents with personality changes even in case of large tumours $[4,9]$. In our case, though not fully personality change, but some amount of change in behavior and attitude was seen, which mimicked childhood depressive disorder. High incidence of increased Intracranial pressure has been reported to be associated with thalamic tumour [7]. Our case had typical symptoms and signs of 
vomiting, dizziness, unsteady gait, sleep walking, somnolence. Possible fiber tracts involvement and Sensory dysfunction resulting from involvement of ventral nuclei [10], were absent in our case.

BLTG presents with characteristics radiological findings. On CT scan brain it appears isodense, but mass effect is difficult to elicit. MRI brain is the best radiological investigation, it provides very detailed information pertaining to the extent (unilateral or bilateral), growth character (focal or infiltrative) and potentially tumour type and grade (from Juvenile Pilocytic Astrocytomas through WHO Grade I to II). In MRI lesion appears hypo to isointense on T1 weighted images and hyperintense on T2 weighted MRI $[3,12]$ and respecting the grey white boundaries and confining to thalamus only. Contrast enhancement is seen only in high grade BLTG, as in our case patchy area of enhancement was seen.

MR spectroscopy is helpful in differentiating bilateral thalamic gliomas from other lesions5. It shows high creatinine and choline peak (creatinine peak greater than choline peak) with decreased peak of NAA, suggestive of higher cellular metabolism (creatinine peak) and higher proliferative potential (choline peak [3]). In our case, increased choline peak and reduction of NAA was noted, which is also suggestive of high grade tumours, (where choline peak is greater than creatinine peak), unlike low grade glioma, where creatinine peak is greater than choline peak. In both high and low grade glioma, reduction of NAA peak is suggestive of neuronal loss.

BLTG are difficult to operate tumours, they are best diagnosed and treated at major medical centres. Biopsy of tumour using minimally techniques like streotactic needle or endoscopic surgery can be done by experienced neurosurgeon. In case of large tumours causing mass effect, debulking is mostly suggested, but that again, may not give satisfactory result. As surgery is often difficult, radiation or chemotherapy were tried but the role of such adjuvant therapies remains unclear $[2-4,8]$. Our case has been referred to neurosurgery department for better management.

\section{Conclusion}

Thalamic glioma is one of the pediatric brain tumour with poor outcome. Bilateral thalamic glioma, as seen on MRI, and biopsy proven Grade III or IV tumour are poor prognostic indicators. However, primary goal in treatment of patients with thalamic glioma is improvement in symptomatology and survival as long as possible. Our case is a peculiar one, because of its atypical symp- tomatology and predominant psychiatric menifestations in later stage. Therefore, detailed evaluation of such cases are required so to have early diagnose and prompt intervention.

\section{Bibliography}

1. A Gupta., et al. "Pediatric thalamic gliomas. An updated review". Archives of Pathology and Laboratory Medicine 141 (2017): 1316-1323.

2. Behari S., et al. "Bilateral astrocytomas: Clinicoradiological characteristics and endoscopic management". Pan Arab Journal of Neurosurgery 13 (2009): 98-103.

3. DK Rajput., et al. "Bilateral thalamicglioma in a 6year-old child". Journal of Pediatric Neurosciences 5.1 (2010): 45-48.

4. Di Rocco C and Iannelli A. "Bilateral thalamic tumors in children". Child's Nervous System 18 (2002): 440-444.

5. Douis H., et al. "Bilateral thalamic glioma". Archives of Neurology 65 (2008): 1666-1667.

6. E Ganewatte., et al. "A case report on 1-year follow up of bilateral thalamic glioma”. Neuroradiology Journal 28.6 (2015): 584-586.

7. GD Partlow., et al. "Bilateral thalamic glioma: review of eight cases with personality change and mental deterioration". American Journal of Neuroradiology 13 (1992): 1225-1230.

8. G Menon., et al. "Bilateral thalamic glioma: report of four cases and review of literature". Journal of Pediatric Neurosciences 1.2 (2006): 66-69.

9. H Hirano., et al. "Nagata, Bilateral thalamic glioma: case report”. Neuroradiology 42 (2000): 732-734.

10. Helle TL and Conley FK. "Hemorrage associated with meningioma: A case report and review of literature". Journal of Neurology, Neurosurgery, and Psychiatry 43 (1980): 725-729.

11. NA Wani., et al. "Bilateral thalamic glioma". Journal of Geriatric Mental Health 2 (2015): 50-52.

12. X Niu., et al. "Prognostic factors for the survival outcome of bilateral thalamic glioma: an integrated survival analysis". World Neurosurgery 110 (2018): e222-e230.

\section{Volume 2 Issue 8 August 2019 \\ (C) All rights are reserved by Monu Doley and H Anjuman Choudhury.}

